lung secondary to atelectasis or after lobectomy. Partial bronchial obstruction from any cause may lead to air trapping. The pulmonary vasculature may be the site of congenital anomalies, or may be occluded by an embolus or thrombus. Occasionally, diminished radiolucency of the opposite lung fields from pleural thickening may cause confusion. Only when these possibilities have been eliminated can a diagnosis of pulmonary hypoplasia be made.

The characteristic radiographic signs are increased transradiancy of a lung or lobe, the affected part being either of normal size or somewhat reduced. The adjacent hilar vascular shadows are small. Screening shows that the volume of the hypoplastic lung remains virtually unchanged during respiration, and that there is a tendency for the mediastinum to move towards the affected side during inspiration. The bronchographic signs are variable and include spidery bronchi, poor peripheral filling, and irregular areas of bronchial dilatation. Angiography confirms the diminished blood flow with narrowing of the pulmonary artery and its branches. Respiratory function tests indicate airways obstruction, increased residual volume, and impaired gas mixing in the hypertranslucent lung. ${ }^{7}$

The pathogenesis of pulmonary hypoplasia is still unclear. ${ }^{8}$ Most arguments are concerned with whether it is the blood vessels or the air passages that are primarily at fault-a congenital vascular defect or retarded development after a bronchopulmonary infection. Certainly, a history of a severe respiratory infection in infancy or childhood is often obtained, and the balance of evidence seems to favour more and more a failure of the immature lung to develop fully after such an infection as the most probable cause.

Treatment is usually not necessary, other than antibiotic therapy for pulmonary infections. Associated bronchiectasis may require surgical excision if it gives rise to recurrent infections or haemoptyses.

\section{New Aspects of Human Genetics}

It is a tribute to the vitality of human genetics that a second number of the British Medical Bulletin has been allotted to this topic after an interval of seven years. A comparison of the two numbers ${ }^{12}$ affords a useful review of some of the main advances in this time.

Two autosomal trisomies other than Down's syndrome (G trisomy) have now been clearly delineated, clinically and cytogenetically. These are Edwards's syndrome (E trisomy) and Patau's syndrome (D trisomy), which have incidences of the order of 1 in 5,000 and 1 in 9,000 live births respectively, whereas the incidence at birth of Down's syndrome is about 1 in 700. The E chromosome involved in Edwards's syndrome is probably 18, the $\mathrm{D}$ chromosome in Patau's probably 13. One syndrome due to a structural anomaly, the cri-duchat syndrome, due to a deletion of the short arm of chromosome 5, is now also clearly delineated. Among the sex chromosome anomalies the remarkable XYY (extra Y) syndrome has been added to the XO (Turner's), the XXY (Klinefelter's) and the XXX syndrome. All but Turner's syndrome are common at birth, with incidences of 1 to 2 per 1,000 live births of the appropriate sex. Study of the chromosomes of foetuses aborted in the first trimester has now shown that infants with chromosome anomalies surviving to term are only the residue of a much larger group of abnormal zygotes. About half the spontaneous abortions in the first three months of pregnancy may have chromosome anomalies. Two of the commonest, present in perhaps $1 \%$ of zygotes, are triploidy with 69 chromosomes present, and an E trisomy which is probably of chromosome 16. Except in mosaics these appear almost always to cause spontaneous abortion.

The XO genotype appears to have the same order of zygote frequency, with only a small minority surviving to term. G and $\mathrm{D}$ trisomies are also not uncommon in abortions. In contrast the sex chromosome trisomies do not appear to predispose to spontaneous abortion.

In biochemical genetics one of the most interesting developments is the finding that multiple allelism-that is, the presence of numerous alternative genes at one gene locus-is a common situation. An excellent example is provided by the human haemoglobins, for many of which the amino-acid sequences have been worked out in detail. Amino-acid substitution can in most cases be interpreted as due to a single base change. Some amino-acid substitutions would be expected to, and do, make the haemoglobin molecule unstable, and so give rise to a congenital haemolytic anaemia. Some substitutions in the pocket of peptide containing the haem molecule cause methaemoglobinaemia. In the thalassaemias there is a failure in synthesis of either the $\alpha$ or the $\beta$ chain of the haemoglobin molecule, but any $\alpha$ or $\beta$ peptide that is synthesized is normal in composition. These could possibly be examples of "controller" gene diseases, but there are several other theoretical ways in which messenger-R.N.A. synthesis could be retarded.

It is now known that in addition to the series of inborn errors of amino-acid metabolism due to a failure of synthesis of enzymes concerned in metabolic pathways-exemplified by classical phenylketonuria-a second series arise from deficiencies in enzymes concerned in amino-acid transport. Two types of transport of amino-acids appear to exist ; one which has a capacity well above the usual concentration of the amino-acid in plasma and which is usually shared by a group of related amino-acids; and one which has a capacity equivalent to the usual plasma concentration of its solute and is usually very specific. Deficiencies of the second mechanism may cause severe disease.

Work on mapping the human autosomes is proceeding steadily with the help of computer programming. Two new methods, however, are being developed which will enable linkage groups to be assigned to individual chromosomes. One depends on the linkage of genes to chromosomes marked by morphological peculiarities. The first example is the linkage of the gene locus for the Duffy blood type to chromosome 1 by means of the secondary constriction present in a proportion of these chromosomes. The other new method depends on the use of mouse-man hybrid cells cultured in vitro. As such hybrid cell cultures divide the human chromosomes gradually drop out unless they are supplying a gene product needed by a mouse cell which has been selected for having a specific enzymatic deficiency. In this way the gene locus producing thymidine kinase has been shown very probably to be present on chromosome 17 .

The field of polygenic inheritance was discussed in the earlier volume, mostly in relation to normal characteristics such as stature, general intelligence, blood pressure, and finger ridge counts. In this volume it is discussed in relation to a number of common malformations and diseases. The family patterns shown by such common conditions suggest that the genetic predisposition to them is polygenic with a threshold.

1 British Medical Bulletin, 1961, 17, No. 3.

2 British Medical Bulletin, 1969, 25, No. 1. 
The concept is an old one, and was first put forward by Karl Pearson some 60 years ago, not long after the principles of Mendel's work were being rediscovered. Heritability estimates on this assumption are often surprisingly high, and this is of importance for the planning of research into the aetiology of these conditions.

\section{Anencephalus and Spina Bifida}

It is an indication of our increasing interest in these malformations that in the short time since anencephalus and spina bifida were last discussed in these columns ${ }^{1}$ several new reports are worth noting. A survey by $\mathrm{K}$. M. Laurence, C. O. Carter, and P. A. David ${ }^{2}$ of over 100,000 births in the Vale of Glamorgan and the mining valleys of South Wales during the years 1956-62 provided details of 364 cases of anencephaly (some of them with spina bifida also) and 425 cases of spina bifida without anencephaly. There were also 46 cases of hydrocephalus not associated with spina bifida, but these will not be considered further because they appear to be quite distinct aetiologically from the other two malformations.

The spina bifida rate $(4.13$ per 1,000$)$ was the highest ever recorded in a population, and the anencephalic rate ( 3.54 per $1,000)$ has been exceeded only once in a population survey (in Northern Ireland ${ }^{3}$ ). There were no significant fluctuations in the annual rates of these malformations, but there were some interesting regional variations, the incidence being highest in the east of the area and lowest in the west. This pattern contrasts sharply with that shown by the British Isles as a whole, where there is a marked downward gradient from west to east. The authors could not account for local variations in incidence by differences in geological formation, background radioactivity, or ethnic, sociological, or economic characteristics. The incidence was higher in urban than in rural areas, but within urban areas it did not seem to be related to population density.

A second paper by the same authors ${ }^{4}$ confirmed an earlier report from Birmingham ${ }^{5}$ that the incidence of both malformations is greater among first-born and among the offspring of young and old mothers. Paternal age seems to have no independent effect. The distribution of $A B O$ and rhesus blood groups among the mothers was no different from that of the general population of the area. In South Wales, as in Birmingham, there was no social class gradient in the incidence of anencephaly. This is in contrast with experience in Scotland, where the families of unskilled manual workers have an incidence five times that of families of the professional and managerial classes. ${ }^{\circ}$

1. British Medical Fournal, 1968, 1, 660. Preventive and Social Medicine, 1968, 22, 146.

- Stevenson, A. C., and Warnock, H. A., Annals of Human Genetics, 1959, 23, 382.

- Carter, C. O., David, P. A., and Laurence, K. M., fournal of Medical Genetics, 1968, 5, 81 .

- Record, R. G., and McKeown, T., British fournal of Social Medicine, 1949, 3, 183 .

- Edwards, J. H., British Fournal of Preventive and Social Medicine,

1958, 12, 115. Medicine and Child Neurology, 1968, 10, Suppl. No. 15, p. 6.

- Record, R. G., and McKeown, T., British Fournal of Social Medicine, $1950,4,217$.

- Yen, S., and MacMahon, B., Lancet, 1968, 2, 623.

10 Carter, C. O., and Fraser Roberts, J. A., Lancet, 1967, 1, 306.

1 Record, R. G., and McKeown, T., Annals of Eugenics, 1951, 15, 285.
The South Wales investigation, in common with most previous studies, provided evidence of familial concentration of these malformations. The incidence of anencephaly or spina bifida among the sibs of affected infants was $5.2 \%$, which is nearly seven times the figure for the general population. In Liverpool the incidence among sibs (4.2\%) was also between six and seven times the general population value, ${ }^{7}$ and in Birmingham, where these defects occur rather less frequently, the same ratio between incidence in sibs and population was observed. ${ }^{8}$ It seems then that the risk of recurrence is greater in some places than others and is related to the local incidence in the population. It has also been shown that in the same locality the risk of recurrence may vary with time and keeps in step with secular variations in the incidence among the general population. ${ }^{9}$ The malformation rate is greater for infants who have two previous sibs affected than for those with only one ${ }^{10}$; in such families the risk to subsequent sibs may be as high as 1 in 10 .

The South Wales data on twins are consistent with earlier reports on the rarity with which both members of a monozygous pair are affected. ${ }^{11}$ There were 13 pairs of twins, of which 9 were of the same sex. This suggests a normal representation of monozygous sets, but there was no instance of the defect in both twins. There was, however, one set of triplets in which both the female members were anencephalic, the third member of the set, a boy, being normal.

A useful analysis of a large twin series collected by S. Yen and B. MacMahon has recently been published.' From the records of 1,793 cases of anencephalus and spina bifida born in hospitals in Rhode Island and Boston, they obtained data on 30 pairs, of which 23 were of like sex. In none of these were both members affected. By adding six other series from the literature to their own data they raised the total to 108 twin pairs. In every case only one member of the pair was affected. The fact that $73 \%$ of the pairs were of like sex suggested that there had been no selective loss of monozygous pairs. This evidence, coupled with that of a number of isolated reports, seems to leave no doubt that both twins rarely suffer from these malformations. In fact concordance is such an uncommon event that if a member of a twin pregnancy is affected the co-twin is less likely to be malformed than is a subsequent sib. Yen and MacMahon explored the possibility that this is due to the early abortion of twin pregnancies in which both members are affected, but found little supporting evidence.

We now know much more about anencephalus and spina bifida than we did 20 years ago, and various interpretations of the facts have been put forward. Few now believe that these malformations can be explained in simple genetical terms, and some geneticists now think that a polygenic mode of inheritance is responsible. But to accommodate the very strong body of evidence indicative of non-genetic aetiological influences they accept that the manifestation of these genes can be influenced by the intrauterine environment ${ }^{4}$ and that genetic mechanisms alone cannot explain the marked national variations in incidence shown for example by Northern Ireland, Wales, Scotland, England, and France. ${ }^{2}$ The familial evidence so far available is equivocal, but a careful unbiased study of half-sibs and of second- and third-degree relatives could be decisive and is badly needed. At present the most revealing evidence has come from twin studies. Unless this evidence has been seriously misinterpreted, the only genetic hypotheses with which it is compatible are, as Yen and MacMahon have pointed out, ones involving low 